\title{
Thermal pure quantum matrix product states recovering a volume law entanglement
}

\author{
Atsushi Iwaki, ${ }^{*}$ Akira Shimizu, and Chisa Hotta ${ }^{\dagger}$ \\ Department of Basic Science, The University of Tokyo, Meguro-ku, Tokyo 153-8902, Japan \\ and Komaba Institute for Science, The University of Tokyo, Meguro-ku, Tokyo 153-8902, Japan
}

(Received 18 May 2020; revised 23 April 2021; accepted 30 April 2021; published 25 May 2021)

\begin{abstract}
We propose a way to construct a thermal pure quantum matrix product state (TPQ-MPS) that can simulate finite-temperature quantum many-body systems with a minimal numerical cost comparable to the matrix product algorithm for the ground state. The MPS was originally designed for the wave function with area law entanglement. However, by attaching the auxiliary sites to the edges of the random matrix product state, we find that the degree of entanglement is automatically tuned so as to recover the volume law of the entanglement entropy that characterizes the TPQ state. The finite-temperature physical quantities of the transverse Ising and the spin-1/2 Heisenberg chains evaluated by a TPQ-MPS show excellent agreement even for bond dimension $\sim 10-20$ with those of the exact results.
\end{abstract}

DOI: 10.1103/PhysRevResearch.3.L022015

Introduction. Finding a good description of typical wave functions of quantum many-body states at finite temperature has been a challenge in condensed matter theory. Traditional quantum mechanical representation of equilibrium states relies on the density operators of Gibbs' ensembles, which are classical mixtures of an exponentially large number of pure quantum states. This construction does not allow us to simulate sufficiently large quantum systems of physical interest, since the available numerical devices, e.g., stochastic quantum Monte Carlo (QMC) methods or some size-free methods [1-4], are limited.

The key conceptual development against the ensemble physics is the typicality [5-10]; there exists $a$ single thermal pure quantum (TPQ) state that solely represents the thermal equilibrium [11-17]. It then happens that for the description of any of the equilibrium quantum states, one can choose arbitrary degrees of the classical mixture, from the purity-[1/( $\left.\left.e^{\Theta(N)}\right)\right]$ ensemble to the purity-1 TPQ state. Let us consider a size- $N$ system with a Hamiltonian $\mathcal{H}$ and its subsystem A of size $n$. Since the equilibrium state at inverse temperature $\beta$ can be described equivalently by the TPQ state $|\psi\rangle_{\beta}$ and by the Gibbs state, the local observables $\mathcal{O}_{A}$ also fulfill

$$
{ }_{\beta}\left\langle\psi\left|\mathcal{O}_{A}\right| \psi\right\rangle_{\beta}=\operatorname{Tr}\left(\mathcal{O}_{A} e^{-\beta \mathcal{H}}\right) / \operatorname{Tr}\left(e^{-\beta \mathcal{H}}\right) .
$$

This directly indicates the equality of the local density matrix and the local canonical ensemble, $\rho_{n}\left(|\psi\rangle_{\beta}\right)=$

\footnotetext{
*iwaki-atsushi413@g.ecc.u-tokyo.ac.jp

${ }^{\dagger}$ chisa@phys.c.u-tokyo.ac.jp
}

Published by the American Physical Society under the terms of the Creative Commons Attribution 4.0 International license. Further distribution of this work must maintain attribution to the author(s) and the published article's title, journal citation, and DOI.
$\operatorname{Tr}_{\bar{A}}\left(e^{-\beta \mathcal{H}}\right) / \operatorname{Tr}\left(e^{-\beta \mathcal{H}}\right)$. Accordingly, the von Neumann entropy $S_{n}=-\operatorname{Tr}\left(\rho_{n} \ln \rho_{n}\right)$ obtained by the reduced density operator $\rho_{n}$ of the subsystem A becomes the entanglement entropy (EE) of a TPQ state and is related to thermodynamic entropy density $s_{t h}$ as [18]

$$
S_{n} / n=s_{t h} \quad(1 \ll n \ll N) .
$$

The EE of the TPQ state thus needs to fulfill the volume law, and indeed, in a similar context, the Page curve of the second Renyi entropy in a finite open boundary system is observed in the exact TPQ state [19].

Practically, however, constructing such an exact TPQ state, which we call a full-TPQ state $[11,15,16]$, requires a cost only slightly smaller than the conventional finite-temperature diagonalization methods [20-22] and is available only up to $\sim 2 N$ of that of the Gibbs state. As for the ground state, the approximate forms of the pure wave functions are established by the density matrix renormalization group (DMRG) or matrix product states (MPSs) [23-25]. However, their application to the TPQ state has never been tested because the MPS description has an area law entanglement by construction [26,27] and is apparently unsuitable for the finite-temperature case, where the entanglement blows up massively.

In this Letter, we show that the TPQ-MPS is realized by attaching appropriate auxiliary sites at both edges of the system, which work as an entanglement bath and make the system highly entangled. By successively operating the Hamiltonian to the random matrix product state (RMPS) with the auxiliaries, the MPS is annealed down to lower temperature, where we find that the system recovers the volume law entanglement when measured from the system center toward the very edges. We demonstrate that the TPQ-MPS wave functions give an accurate evaluation of the physical quantities in typical quantum spin models without taking the ensemble average. The computational cost is significantly reduced to that of the MPS 

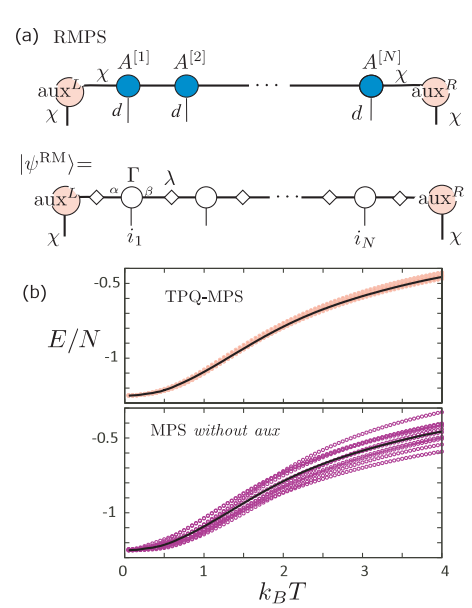
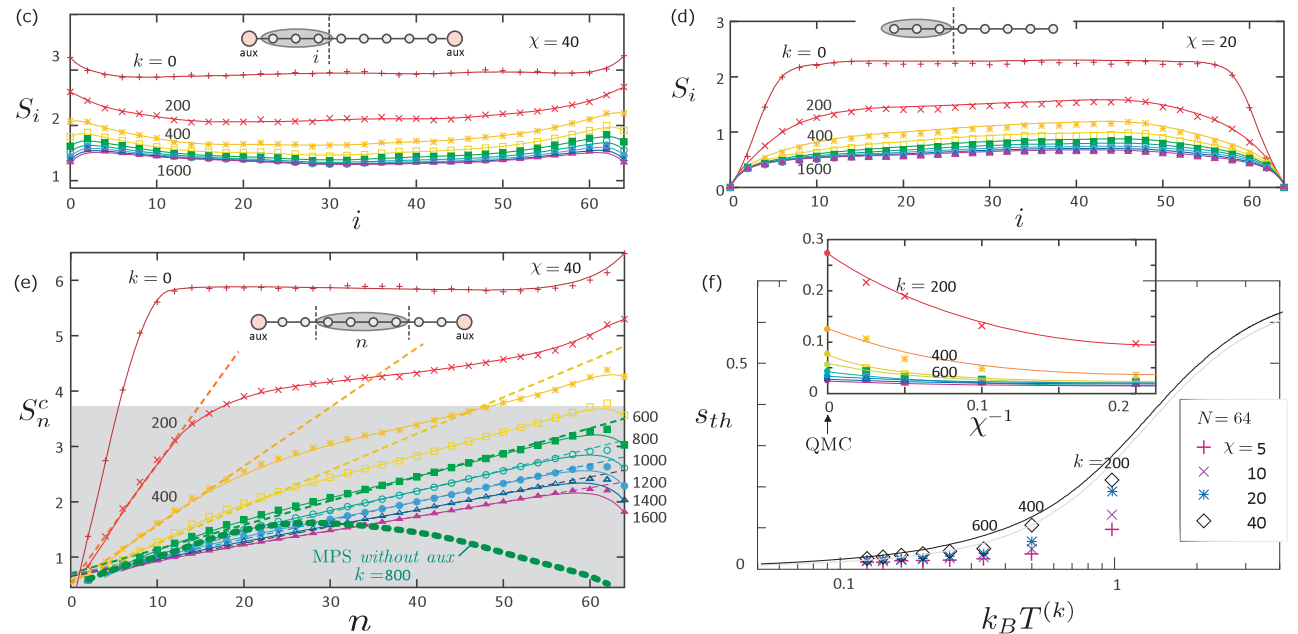

FIG. 1. (a) Schematic representation of the RMPS in Eq. (3). Two auxiliary sites (pink circles) are connected to the two open edges of the system, which have no physical interactions with the main system. The lower panel is the canonical form of the TPQ-MPS $\left|\psi^{\mathrm{RM}}\right\rangle$. For the starting point of mTPQ $(k=0)$ we take $\Gamma_{\alpha_{m} \beta_{m}}^{[m] i_{m}}$ of $\chi \times \chi \times d$ with a Gaussian distribution, $\lambda_{i}=1$, and aux $\alpha_{X}^{X} \beta$ of $X=L, R$ as the $\chi \times \chi$ unit matrices, which gives the canonical form of the RMPS. (b) Energy density of the transverse Ising model plotted independently for ten mTPQ runs. Upper and lower panels are those of TPQ-MPS and MPS without auxiliaries (aux) with $N=16$ and $\chi=40$. (c) and (d) Entanglement entropy $S_{i}=-\operatorname{Tr}\left(\hat{\rho}_{i} \ln \hat{\rho}_{i}\right)$ of the TPQ-MPS and MPS without auxiliaries, when the $N=64$ system is divided at bond $i=1 \sim N$. The $k$ th mTPQ state of the transverse Ising model with fixed $\chi=40,20$ is shown. (e) Entanglement entropy $S_{n}^{c}=-\operatorname{Tr}\left(\hat{\rho}_{n} \ln \hat{\rho}_{n}\right)$ of the TPQ-MPS when dividing the $N=64$ system and picking up the $n$ sites from the center. Dashed lines are the linear fits whose slope gives the thermodynamic entropy $s_{t h}$. The bold green dashed line is $S_{n}^{c}$ obtained without auxiliaries for $k=800$. The shade marks $S_{n}^{c} \lesssim \ln \chi$ allowed for the standard MPS for $\chi=40$. (f) Slope of $S_{n}^{c}$ as a function of $k_{B} T^{(k)}$ for $k=200-1600$ in the mTPQ calculation for $\chi=5,10,20,40$ and $N=64$. Solid black and gray lines are $s_{t h}$ obtained by the QMC results at $N=64$ and 32, respectively. Inset: $\chi^{-1}$ dependence of the data where the circles at the starting point are the QMC results for the corresponding temperature.

of the ground state, and the accessible system size increases to $N \gtrsim 100$.

So far, MPS methods at finite temperature such as minimally entangled typical thermal states (METTSs) [28,29] and matrix product purification (MPP) [30-32], both with intermediate degrees of purity, have relied on some sort of "ensemble" averages to compensate for the low entanglement properties of the MPS. The METTS starts from the minimally entangled product state, which requires a large number of sampling averages, $\gtrsim 100$ [33]. MPP adopts an extra $N$ ancillary systems, which are traced out to obtain the mixed state of the system [34]; this expression is mathematically redundant since they replace a mixed state in an irreducible representation in the minimum Hilbert space of dimension $d^{N}$ by a reducible representation in a space of dimension $d^{2 N}$, and does not save our numerical cost by itself.

Random initial state. We consider a one-dimensional (1D) lattice system consisting of $N$ sites with open boundary conditions (OBCs), where each site hosts $d$-dimensional degrees of freedom, and two auxiliaries attached at both edges having $\chi$ dimensions. The RMPS of such a system shown in Fig. 1(a) is given as [35]

$$
\begin{aligned}
\left|\psi^{\mathrm{RM}}\right\rangle= & \sum_{\alpha_{L}\left\{i_{n}\right\} \alpha_{R}}\left\langle\operatorname{aux}_{\alpha_{L}}^{L}\left|A^{[1] i_{1}} \cdots A^{[N] i_{N}}\right| \operatorname{aux}_{\alpha_{R}}^{R}\right\rangle \\
& \left|\alpha_{L}, i_{1} \cdots i_{N}, \alpha_{R}\right\rangle,
\end{aligned}
$$

where the $A^{[m] i_{m}}$ is the $d \chi^{2}$ matrix on the $m$ th site with $i_{m}=1, \ldots, d$ and is explicitly given using the $d \chi \times d \chi$ random unitary matrix $U$ as $A_{\alpha \beta}^{[m] i_{m}}=U_{(i, \alpha)(1, \beta)}$ or $U_{(1, \alpha)(i, \beta)}$, which fulfill the left or right canonical form, respectively. Here, $\left|\operatorname{aux}_{\alpha_{L / R}}^{L / R}\right\rangle$ is the right or left auxiliary state with $\alpha_{L / R}=1$ to $\chi$. This RMPS reproduces the physical quantities at $T=\infty$ with a variance of order $\chi^{-2}$, which can be shown analytically as follows. Taking $A$ on the lefthand side or right-hand side of a one-site operator $\hat{\mathcal{O}}_{i}$ as the left or right canonical form, we have $\left\langle\psi^{\mathrm{RM}} \mid \psi^{\mathrm{RM}}\right\rangle=$ $\chi$. By taking account of the formula for the random average, $\overline{U_{i j} U_{k l}^{*}}=\delta_{i j} \delta_{k l} /(\chi d)$ and $\overline{U_{i j} U_{i k}^{*} U_{l m} U_{l n}^{*}}=\left[\delta_{j k} \delta_{m n}+\right.$ $\left.\delta_{i l} \delta_{j n} \delta_{m k}-\left(\delta_{i l} \delta_{j k} \delta_{m n}+\delta_{j n} \delta_{m k}\right) /(\chi d)\right] /\left(\chi^{2} d^{2}-1\right)$, we have the expectation values $\left\langle\psi^{\mathrm{RM}}|\cdots| \psi^{\mathrm{RM}}\right\rangle / \chi \equiv\langle\cdots\rangle$ as

$$
\overline{\left\langle\hat{\mathcal{O}}_{i}\right\rangle}=\sum_{i, j, \alpha, \beta} \frac{1}{\chi^{2} d} \delta_{i j}\left\langle j\left|\hat{\mathcal{O}}_{i}\right| i\right\rangle=\left\langle\mathcal{O}_{i}\right\rangle_{\infty},
$$

with $\left\langle\mathcal{O}_{i}\right\rangle_{\infty} \equiv d^{-1} \operatorname{Tr} \mathcal{O}_{i}$, and its variance as

$$
\overline{\left\langle\hat{\mathcal{O}}_{i}\right\rangle^{2}}-\left(\overline{\left\langle\hat{\mathcal{O}}_{i}\right\rangle}\right)^{2}=\frac{d-1}{\chi^{2} d^{2}-1}\left(\left\langle\hat{\mathcal{O}}_{i}^{2}\right\rangle_{\infty}-\left\langle\hat{\mathcal{O}}_{i}\right\rangle_{\infty}^{2}\right) .
$$

The typicality of the RMPS is studied and confirmed numerically in a similar context $[35,36]$, followed by several proposals to stochastically construct microcanonical and canonical ensembles of the RMPS [37-39].

In our work, the RMPS is constructed not by using Eq. (3) but by preparing a tensor $\Gamma_{\alpha_{m} \beta_{m}}^{[m] i_{m}}(\in \mathbb{C})$ of bond dimension $\chi$, whose elements follow the Gaussian distribution [see Fig. 1(a)]. It can be straightforwardly shown that after transforming Eq. (3) into the canonical form by the successive Schmidt decomposition [40,41], the obtained matrices also form an equivalent RMPS. 
mTPQ-MPS method. The initial state $(k=0)$ is taken as the aforementioned RMPS with bond dimension $\chi$, where we take auxiliaries of $\chi \times \chi$ attached at both edges as a unit matrix $\hat{I}$ in the first place. The RMPS successively generates a series of un-normalized microcanonical TPQ (mTPQ) states $k=0,1,2, \ldots$ as $[15]$

$$
|k\rangle=(l-\hat{h})^{k}\left|\psi^{\mathrm{RM}}\right\rangle,
$$

where $\hat{h}$ is the Hamiltonian divided by $N$ and $l$ is a parameter larger than the maximum eigenenergy, which is necessary to generate a sharp microcanonical energy distribution (see Ref. [15]). Here, $|k\rangle$ is the TPQ state at a temperature $k_{B} T^{(k)}=$ $N\left(l-u_{k}\right) / 2 k[42]$ with energy $u_{k}=\langle k|\hat{h}| k\rangle /\langle k \mid k\rangle$. In our algorithm, $(l-\hat{h})$ is represented by a matrix product operator (MPO) of bond dimension $D$ that depends on $\hat{h}$, and applying this MPO at each step to $|k\rangle$ multiplies the matrix dimension to $D \chi$. Before truncating the dimension of the enlarged matrix down to $\chi$, we transform the MPS to its canonical form including auxiliary sites in order to minimize the truncation error [43]. The process is repeated until $k_{B} T^{(k)}$ reaches a low enough temperature $\beta_{\max }^{-1}$. The effective bond dimension $\chi_{i}^{\text {eff }}(i=0, N)$, which is the number of finite eigenvalues of the Schmidt decomposition $\lambda_{i}$ on the $i$ th bond, can change automatically within $1 \leqslant \chi_{i}^{\text {eff }} \leqslant \chi$.

We consider an operator $\hat{A}$ that can be described by a low-order polynomial of local observables. At each step, such $\hat{A}$ is evaluated and stored; $\langle\hat{A}\rangle=\langle k|\hat{A}| k\rangle /\langle k \mid k\rangle$ is the physical quantities at $k_{B} T^{(k)}$. Instead of directly adopting this form, one can generate the physical quantities for arbitrary temperatures $\beta^{-1} \gtrsim \beta_{\max }^{-1}$ by the canonical summation as (see Ref. [16])

$\langle\beta|\hat{A}| \beta\rangle=e^{-\beta N l} \sum_{k}\left\{\frac{(N \beta)^{2 k}}{(2 k) !}\langle k|\hat{A}| k\rangle+\frac{(N \beta)^{2 k+1}}{(2 k+1) !}\langle k|\hat{A}| k+1\rangle\right\}$.

The quality of the TPQ-MPS is tested by the comparison of $\langle\hat{A}\rangle_{\beta}=\langle\beta|\hat{A}| \beta\rangle /\langle\beta \mid \beta\rangle$ with the exact or nearly exact solution by exact diagonalization (ED), QMC, or quantum transfer matrix (QTM) [44,45] methods [46].

The required number of random averages of $\mathrm{MTPQ}$ runs $N_{\text {ran }}$ is as small as in the full TPQ that uses the full Hilbert space, e.g., typically less than 5 for $N \gtrsim 32$. This can be seen from the benchmark results in Fig. 1(b): When performing ten mTPQ runs with and without auxiliaries, the variance of the former turned out to be small by orders of magnitude, particularly at higher temperature. The variance of TPQ-MPS decreases at lower $T$, contrarily to the full TPQ. The higher performance despite its being an approximate method is possibly because the basis in the MPS construction is optimally biased at low temperature to those favoring the low-energy states. The difference from the original TPQ also lies in that the range of mTPQ temperature $k_{B} T^{(k)}$ depends much on $l$. Usually, starting from smaller $l$ will accelerate the convergence, and we reach the same temperature with smaller $k_{\max }$. However, because of finite $\chi$, the starting temperature at $k \sim 1$ for $l \ll \Theta(h)$ is kept to $k_{B} T \lesssim \Theta(h)$ (h being the typical local energy scale of the Hamiltonian), in which case the canonical summation becomes inaccurate. In contrast, $l \gtrsim \Theta(10 h)$ sacrifices the low-temperature information, and one needs to set the proper $l$ depending on the models.
Volume law and the auxiliaries. Let us first examine the basic entanglement properties of the TPQ-MPS. Here, we benchmark the 1D transverse Ising chain, $\hat{H}=$ $J \sum_{i=1}^{N-1} \hat{\sigma}_{i}^{z} \hat{\sigma}_{i+1}^{z}-g \sum_{i=1}^{N} \hat{\sigma}_{i}^{x}$, with $\sigma^{z}= \pm 1$, whose MPO has $D=3$, and take $J=g=1$. Figures 1(c) and 1(d) show the EE, $S_{i}=-\operatorname{Tr}\left(\hat{\rho}_{i} \ln \hat{\rho}_{i}\right)$, where $\hat{\rho}_{i}$ is the density matrix when dividing the system into left and right parts at the $i$ th bond. It is known that $S_{i}$ of the TPQ state follows a Page curve [47], which increases linearly from the edges that reflect the volume law and saturates toward the maximum at the center. The standard MPS without auxiliaries in Fig. 1(d) indeed follows a Page-like curve at the edge up to the bond dimension, where $S_{i}$ becomes flat because of the upper bound by the bond dimension $\chi$. Here, the lower temperature or larger $k$ requires the smaller $\chi$. In contrast, in TPQ-MPS, the auxiliaries introduce to the edge a large $S_{0}$ that depends only on the effective bond dimension at the edge, generating a nearly flat $S_{i}$ throughout the system.

However, if we divide the system by setting the subsystem at the center, namely, cutting the two bonds at equal distances from the center, we find physically meaningful entanglement properties. Figure 1(e) is the corresponding EE, $S_{n}^{c}=-\operatorname{Tr}\left(\hat{\rho}_{n} \ln \hat{\rho}_{n}\right)$, as a function of the size $n$ of the subsystem. One finds that $S_{n}^{c}$ increases linearly in $n$ until it saturates to the upper bound. At $k \gtrsim 600$, where the system reaches the temperature $k_{B} T^{(k)} \lesssim 0.33, S_{n}^{c}$ continues to increase up to the very edges of the system.

Entropy. These results indicate that the TPQ-MPS wave function has acquired qualitatively different degrees of freedom in its state space, just by attaching the two auxiliaries. While the upper bound of the EE, $2 \ln \chi$, is only twice as large as the case without auxiliaries, the EE continues to go up until that bound since the MPS does not feel the edge. This is in sharp contrast to the standard MPS, where the entanglement is suppressed toward both edges [Fig. 1(e), bold green dashed line]; although the bound of $S_{n}^{c}$ for the latter was theoretically believed to be $\ln \chi \sim 3.7$ (see shaded region), the realized value is much smaller, $S_{n}^{c} \lesssim 1-1.5$, and behaves linearly only up to $n \sim 10$ near the edges.

Because of this advantage, the present scheme allows the evaluation of the thermodynamic entropy density $s_{t h}$ using Eq. (2): We perform a linear fit of a series of $S_{n}^{c}$ for $N=64$ and for various $\chi$ and compare its slope with $s_{t h}$ obtained by integrating the specific heat of the QMC calculation. As shown in Fig. 1(f), the slope of $S_{n}^{c}$ agrees with $s_{t h}$, asymptotically approaching the QMC data with increasing $\chi$. In principle, we need to take $\chi \sim e^{s_{t h} n / 2}$ to attain a volume law of the subsystem size $n$. This fact is unrelated to size $N$. The TPQ-MPS makes full use of the theoretical bound of $S_{n} \lesssim$ $2 \ln \chi=4-10$ for $\chi=50-100$, which affords a description of the entanglement of the pure state in the standard target temperature range, e.g., $k_{B} T \leqslant J$, that is of physical interest in typical quantum lattice models. By contrast, $S_{n}$ of the usual MPS is unreliable because it is tightly bounded by the peak of the Page curve related to $N$, which is lower than $\ln \chi$.

Benchmarks. Figure 2(a) shows the energy density $e=$ $E / N$ of the transverse Ising model for $N=16$, which is in good agreement with the QMC results with OBCs, where we put together the periodic boundary conditions (PBCs) of the same size. The $N=16$ and 64 cases are also compared 

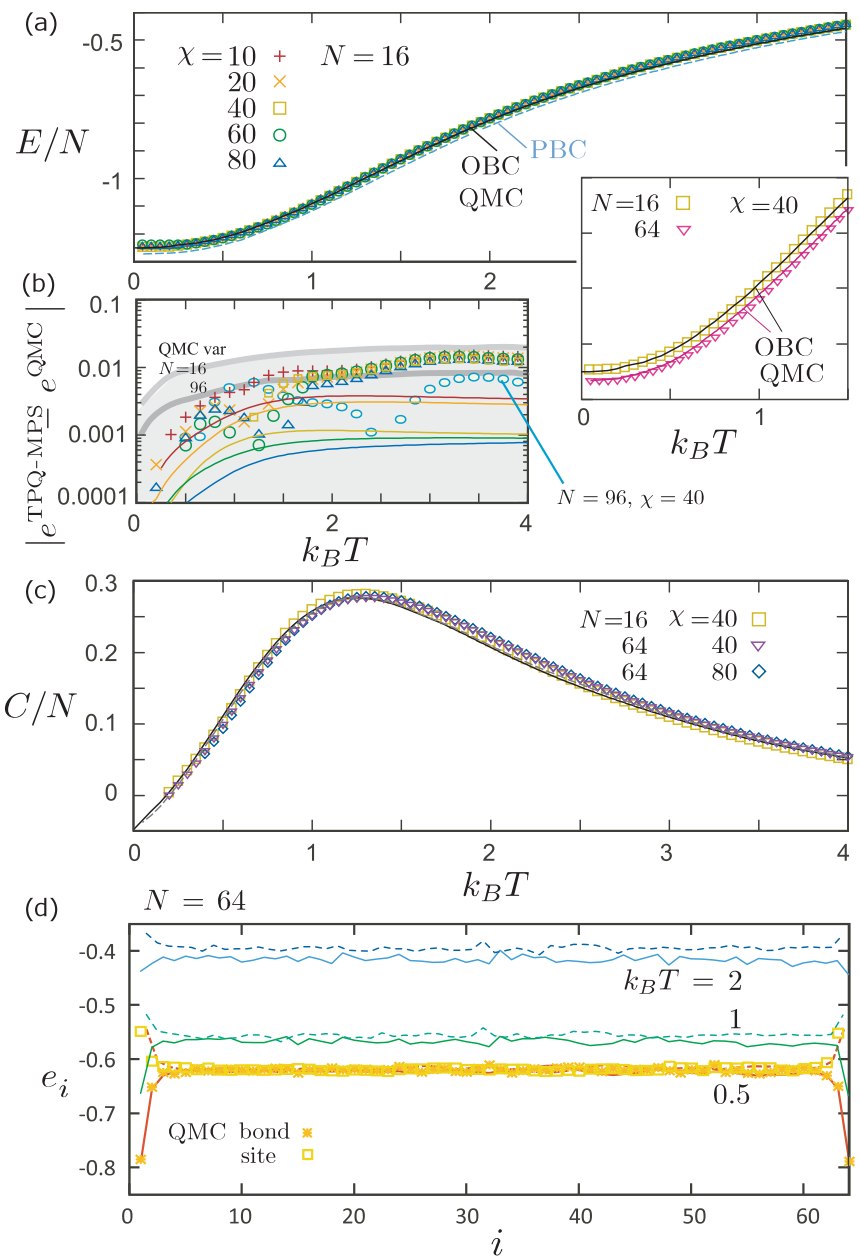

FIG. 2. Comparison of the results of the TPQ-MPS and QMC done by the authors in the transverse Ising model with $J=g=1$. We choose $l=5$ and $k_{\max }=500,1200,2000$ for $N\left(N_{\text {ran }}\right)=16(20)$, 64 (5), 96 (5), respectively, giving $\beta_{\max }^{-1} \sim 0.1$. (a) Energy density $e=E / N$ for $N=16$ with $\chi=5,10,20$ and $l=5$ as a function of $k_{B} T$ compared with the PBC and OBC results from a QMC of the same size. The inset shows the low-temperature part with $(N, \chi)=(16,40)$ and $(64,40)$ with $l=10$ and the corresponding QMC. (b) The energy difference $\left|e^{\mathrm{TPQ}-\mathrm{MPS}}-e^{\mathrm{QMC}}\right|$ from (a) and $N=96, \chi=40$, the variance of the TPQ-MPS average (solid lines) for $N=16, \chi=20-40$, and the variance of the QMC (bold lines above the shaded areas) for 100 independent runs. (c) Specific heat $C / N$ for the same data as (a), and $N=64,96, \chi=40$ with $l=5$ in the inset. QMC-OBC results for the same sizes are given as a solid line. (d) Spatial distribution of the site and bond energies (solid and dashed lines) for $N=64$ and $k_{B} T=0.5,1,2$. QMC-OBC results are shown by symbols for $k_{B} T=0.5$.

in the inset, demonstrating that the finite-size effect is much larger than the difference between the TPQ-MPS and QMC results. Already at $\chi \gtrsim 10$, the error is converged as we see in Fig. 2(b) and is smaller than the variance of the QMC results over 100 independent runs each with 200000 averages, regardless of size $N$ and $\chi$. The specific heat $C / N$ also gives excellent agreement with the QMC results of the same size [see Fig. 2(c)]. Figure 2(d) gives the spatial distribution of the bond and site energies ( $J$ and $g$ terms of the Hamiltonian) for
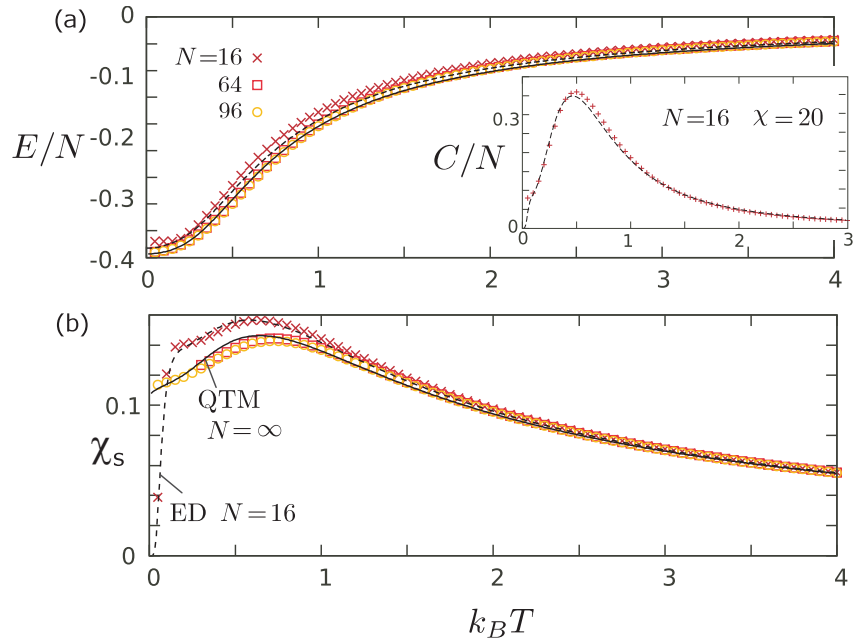

FIG. 3. Physical properties of the Heisenberg model with $J=1$ compared with the $N=16$ ED-OBC and QTM $(N=\infty$ exact solution) ones [45] shown with dashed and solid lines, respectively. We choose $l=1$ and $\beta_{\max }^{-1} \sim 0.03$ with $k_{\max }=500(N=16), 2000$ $(N=64,96)$. (a) Energy density $e=E / N$ and (b) susceptibility $\chi_{s}$ for $N=16,64,96$ with $\chi=20,40,40$ as a function of $k_{B} T$. The inset in (a) shows the specific heat for $N=16$.

several temperatures at $N=64$. They perfectly follow those of OBCs obtained by QMC for $k_{B} T=0.5$ even at the very edges that show downturn or upturn. The variances of mTPQ runs are kept small enough; $10-20$ averages for $N=16$ and 5 for $N=64,96$ in Figs. 2(a)-2(d).

Next, we test our method with the Heisenberg chain described by $\hat{H}=\sum_{i=1}^{N-1} J \hat{s}_{i} \hat{s}_{i+1}$, with $s_{i}^{z}= \pm 1 / 2$. The MPO for this Hamiltonian has $D=5$, which would increase the truncation error. In fact, it is known for the MPS ground state that the model requires much larger $\chi$ compared with the product-type ground state of the transverse Ising model. Figure 3(a) shows $E / N$ and $C / N$ for several system sizes in comparison with the $N=16 \mathrm{ED}$ with $\mathrm{OBCs}$ and the $N=\infty$ exact solution (QTM) [45], which shows good agreement for the same order of $\chi$ as the transverse Ising model. We also plot in Fig. 3(b) the susceptibility $\chi_{s}$ to see how much a well-known logarithmic singularity at the lowest temperature can be traced by the TPQ-MPS. The drop of $\chi_{s}$ at $N=16$ is almost perfectly reproduced already for $\chi=20$. Also, the larger size results are in reasonable agreement with the QTM.

Conclusion. We realized the TPQ-MPS by attaching the edge auxiliaries of dimension $\chi$ to the MPS, showing that the EE of the subsystem cut out from the center continues to show a volume law up to the very edges of the system, particularly at low temperature, by setting the bond dimension $\chi$ to realistically small values. Physical properties are evaluated almost free of random sampling. Such a construction enables the application to higher dimensions, possibly less costly than the previously developed tensor network approaches [48-51].

Acknowledgments. We thank Tsuyoshi Okubo and Hal Tasaki for discussions. The work is supported by JSPS KAKENHI Grants No. JP17K05533, No. JP18H01173, No. JP17K05497, No. JP17H02916, and No. JP19H01810. 
[1] J. Oitmaa, C. Hamer, and W. Zheng, Series Expansion Methods for Strongly Interacting Lattice Models (Cambridge University Press, Cambridge, 2010).

[2] R. F. Bishop, D. J. J. Farnell, S. E. Krüger, J. B. Parkinson, J. Richter, and C. Zeng, High-order coupled cluster method calculations for the ground- and excited-state properties of the spin-half $X X Z$ model, J. Phys.: Condens. Matter 12, 6887 (2000).

[3] C. Hotta and K. Asano, Magnetic susceptibility of quantum spin systems calculated by sine square deformation: Onedimensional, square lattice, and kagome lattice Heisenberg antiferromagnets, Phys. Rev. B 98, 140405(R) (2018).

[4] B. Bernu, L. Pierre, K. Essafi, and L. Messio, Effect of perturbations on the kagome $s=\frac{1}{2}$ antiferromagnet at all temperatures, Phys. Rev. B 101, 140403(R) (2020).

[5] J. von Neumann, Proof of the ergodic theorem and the H-theorem in quantum mechanics, Eur. Phys. J. H 35, 201 (2010).

[6] M. Imada and M. Takahashi, Quantum transfer Monte Carlo method for finite temperature properties and quantum molecular dynamics method for dynamical correlation functions, J. Phys. Soc. Jpn. 55, 3354 (1986).

[7] T. Monnai and A. Sugita, Typical pure states and nonequilibrium processes in quantum many-body systems, J. Phys. Soc. Jpn. 83, 094001 (2014).

[8] S. Popescu, A. J. Short, and A. Winter, Entanglement and the foundations of statistical mechanics, Nat. Phys. 2, 754 (2006).

[9] S. Goldstein, J. L. Lebowitz, R. Tumulka, and N. Zanghì, Canonical Typicality, Phys. Rev. Lett. 96, 050403 (2006).

[10] P. Reimann, Typicality for Generalized Microcanonical Ensembles, Phys. Rev. Lett. 99, 160404 (2007).

[11] A. Hams and H. De Raedt, Fast algorithm for finding the eigenvalue distribution of very large matrices, Phys. Rev. E 62, 4365 (2000).

[12] M. Machida, T. Iitaka, and S. Miyashita, Temperature dependence of ESR intensity for the nanoscale molecular magnet $\mathrm{V}_{15}$, J. Phys. Soc. Jpn. 74, 107 (2005).

[13] M. Machida, T. Iitaka, and S. Miyashita, ESR intensity and the Dzyaloshinsky-Moriya interaction of the nanoscale molecular magnet $\mathrm{V}_{15}$, Phys. Rev. B 86, 224412 (2012).

[14] T. Iitaka and T. Ebisuzaki, Algorithm for Linear Response Functions at Finite Temperatures: Application to ESR Spectrum of $s=\frac{1}{2}$ Antiferromagnet $\mathrm{Cu}$ Benzoate, Phys. Rev. Lett. 90, 047203 (2003).

[15] S. Sugiura and A. Shimizu, Thermal Pure Quantum States at Finite Temperature, Phys. Rev. Lett. 108, 240401 (2012).

[16] S. Sugiura and A. Shimizu, Canonical Thermal Pure Quantum State, Phys. Rev. Lett. 111, 010401 (2013).

[17] M. Hyuga, S. Sugiura, K. Sakai, and A. Shimizu, Thermal pure quantum states of many-particle systems, Phys. Rev. B 90, 121110(R) (2014).

[18] See Supplemental Material at http://link.aps.org/supplemental/ 10.1103/PhysRevResearch.3.L022015 for the proof of Eq. (2) and a detailed explanation regarding this paragraph.

[19] Y. O. Nakagawa, M. Watanabe, H. Fujita, and S. Sugiura, Universality in volume-law entanglement of scrambled pure quantum states, Nat. Commun. 9, 1635 (2018).

[20] J. Jaklič and P. Prelovšek, Lanczos method for the calculation of finite-temperature quantities in correlated systems, Phys. Rev. B 49, 5065(R) (1994).
[21] M. Aichhorn, M. Daghofer, H. G. Evertz, and W. von der Linden, Low-temperature Lanczos method for strongly correlated systems, Phys. Rev. B 67, 161103(R) (2003).

[22] J. Schnack, J. Richter, and R. Steinigeweg, Accuracy of the finite-temperature Lanczos method compared to simple typicality-based estimates, Phys. Rev. Research 2, 013186 (2020).

[23] M. Fannes, B. Nachtergaele, and R. F. Werne, Exact antiferromagnetic ground states of quantum spin chains, Europhys. Lett. 10, 633 (1989).

[24] M. Fannes, B. Nachtergaele, and R. F. Werne, Valence bond states on quantum spin chains as ground states with spectral gap, J. Phys. A: Math. Gen. 24, L185 (1991).

[25] F. Verstraete and J. I. Cirac, Matrix product states represent ground states faithfully, Phys. Rev. B 73, 094423 (2006).

[26] L. Amico, R. Fazio, A. Osterloh, and V. Vedral, Entanglement in many-body systems, Rev. Mod. Phys. 80, 517 (2008).

[27] J. Eisert, M. Cramer, and M. B. Plenio, Colloquium: Area laws for the entanglement entropy, Rev. Mod. Phys. 82, 277 (2010).

[28] S. R. White, Minimally Entangled Typical Quantum States at Finite Temperature, Phys. Rev. Lett. 102, 190601 (2009).

[29] E. M. Stoudenmire and S. R. White, Minimally entangled typical thermal state algorithms, New J. Phys. 12, 055026 (2010).

[30] F. Verstraete, J. J. García-Ripoll, and J. I. Cirac, Matrix Product Density Operators: Simulation of Finite-Temperature and Dissipative Systems, Phys. Rev. Lett. 93, 207204 (2004).

[31] M. Zwolak and G. Vidal, Mixed-State Dynamics in OneDimensional Quantum Lattice Systems: A Time-Dependent Superoperator Renormalization Algorithm, Phys. Rev. Lett. 93, 207205 (2004).

[32] A. E. Feiguin and S. R. White, Finite-temperature density matrix renormalization using an enlarged Hilbert space, Phys. Rev. B 72, 220401(R) (2005).

[33] Regarding the numerical efficiency, the calculation time of METTS for a single $k_{B} T=\beta^{-1}$ scales as $\left(N \chi^{3} \beta\right) \times N_{\tau}$, where the Markov sampling (time step) is typically $N_{\tau} \gtrsim 100$ and $N \chi^{3}$ is the cost of taking a canonical form and truncation with a maximal bond dimension $\chi=50-5000$. The cost of TPQ-MPS to be compared is $\left(N \chi^{3} k_{\max }\right) \times N_{\text {ran }}$, with $k_{\max }=500-2000$ depending on the size and $N_{\text {ran }} \sim 5-20$ that decreases with $N$, which all at once gives the data for the whole temperature range of $k_{B} T=0.01-6$.

[34] H. Umezawa, H. Matsumoto, and M. Tachiki, Thermo Field Dynamics and Condensed States (North Holland, Amsterdam, 1982).

[35] S. Garnerone, T. R. de Oliveira, and P. Zanardi, Typicality in random matrix product states, Phys. Rev. A 81, 032336 (2010).

[36] S. Garnerone, T. R. de Oliveira, S. Haas, and P. Zanardi, Statistical properties of random matrix product states, Phys. Rev. A 82, 052312 (2010).

[37] S. Garnerone and T. R. de Oliveira, Generalized quantum microcanonical ensemble from random matrix product states, Phys. Rev. B 87, 214426 (2013).

[38] S. Garnerone, Pure state thermodynamics with matrix product states, Phys. Rev. B 88, 165140 (2013).

[39] T. Iitaka, Random phase product sate for canonical ensemble, arXiv:2006.14459.

[40] Y.-Y. Shi, L.-M. Duan, and G. Vidal, Classical simulation of quantum many-body systems with a tree tensor network, Phys. Rev. A 74, 022320 (2006). 
[41] G. Vidal, Efficient Classical Simulation of Slightly Entangled Quantum Computations, Phys. Rev. Lett. 91, 147902 (2003).

[42] In the mTPQ algorithm, $k_{B} T^{(k)}=N\left(l-u_{k}\right) / 2 k$ gives a rough estimate of the microcanonical temperature of $\Theta(1 / N)$. Higherorder corrections can be evaluated following Ref. [15].

[43] We define the truncation error as the weight discarded in the Schmidt decomposition. In the transverse Ising model, the maximum truncation error at each $k$ is typically $10^{-4}-10^{-5}$ for $k_{B} T^{(k)} \lesssim 1$ at $N=16$ and decreases by orders of magnitude with both $N$ and $\chi$.

[44] A. Klümper, Thermodynamics of the anisotropic spin-1/2 Heisenberg chain and related quantum chains, Z. Phys. B: Condens. Matter 91, 507 (1993).

[45] A. Klümper, The spin-1/2 Heisenberg chain: thermodynamics, quantum criticality and spin-Peierls exponents, Eur. Phys. J. B 5, 677 (1998).

[46] In the previous TPQ formalism where $\left|\psi^{\mathrm{RM}}\right\rangle$ in Eq. (6) is the random state constructed using the full Hilbert space, the variance of $\langle\hat{A}\rangle_{k}^{\mathrm{TPQ}}$ against the ensemble average
$\langle\hat{A}\rangle=\operatorname{Tr}\left[\hat{\rho}_{k} \hat{A}\right]$ is proved to be as small as of the order of $e^{-\Theta(N)}[15,16]$. Although it is no longer guaranteed, the following demonstration shows comparably accurate results for the TPQ-MPS.

[47] D. N. Page, Average Entropy of a Subsystem, Phys. Rev. Lett. 71, 1291 (1993).

[48] B.-B. Chen, L. Chen, Z. Chen, W. Li, and A. Weichselbaum, Exponential Thermal Tensor Network Approach for Quantum Lattice Models, Phys. Rev. X 8, 031082 (2018).

[49] H. Li, B.-B. Chen, Z. Chen, J. von Delft, A. Weichselbaum, and $\mathrm{W}$. Li, Thermal tensor renormalization group simulations of square-lattice quantum spin models, Phys. Rev. B 100, 045110 (2019).

[50] B. Bruognolo, Z. Zhu, S. R. White, and E. M. Stoudenmire, Matrix product state techniques for two-dimensional systems at finite temperature, arXiv:1705.05578v2.

[51] D.-W. Qu, W. Li, and T. Xiang, Thermal tensor network simulations of the Heisenberg model on the Bethe lattice, Phys. Rev. B 100, 125121 (2019). 